\title{
Influence of Intercropping Legume Fodder Crop Guar with Sesame for Reducing Charcoal-Rot and wilt Disease Complex concerning to its Yield and Yield Components
}

\author{
Nagwa R. Ahmed ${ }^{1}$ and Mohamed M. A. Ibrahim ${ }^{2}$ \\ ${ }^{1}$ Crop Intensification Res. Dept., Field Crops Res. Inst., ARC, Giza, Egypt. \\ ${ }^{2}$ Plant Path. Res. Inst., ARC, Giza, Egypt. \\ Corresponding author:drnagwarefat2014@gmail.com
}

\begin{abstract}
A field trial was conducted at Mallawi Agricultural Research Station, Minia Governorate, ARC, during 2016 and 2017 seasons in a high naturally infested field with charcoal rot and wilt diseases complex, as well as under artificially infestation with charcoal rot and wilt pathogens i.e. Macrophomina phaseolina and Fusarium oxysporum, respectively. The aim of this working to study the effect of intercropping guar with sesame at three planting densities of 100, 67 and 50\% from sole guar and number of once and twice to reduce charcoal rot and wilt diseases complex, and yield and yield components of both crops. All intercropping patterns were significantly decreased disease incidence of charcoal rot and wilt diseases compared to the sole sesame under field and pots conditions. The intercropping $100 \%$ Sesame $+100 \%$ guar was the best pattern to reduce the incidence of charcoal rot \& wilt disease complex in both seasons and pots experiment. Sesame seed yield/fad with guar at $50 \%$ plant density was increased by $32.66,23.27 \%$ and $28.51,18.58 \%$ than those of guar at of $100 \%$ and $67 \%$ in the $1{ }^{\text {st }}$ and 2 nd, seasons, respectively. The highest value of total fresh yield/fad (ton) and total dry yield/fad (ton) of guar with sesame at intercropping pattern 100\% guar were 5.91, 0.79 and 6.69, 0.69 ton/fad in the first and second seasons, respectively. Intercropping 50\% guar with sesame and one cut guar recorded the highest values for LER, net return and MAI (monetary advantage index) in both seasons. Meanwhile, the lowest net return was recorded for intercropping $100 \%$ with two cuts of guar. Our study concluded that intercropping crop guar can be used for the protection of sesame charcoal- rot and wilt disease complex and produced greater seed yield than either crops grown alone.
\end{abstract}

Keywords: guar, sesame, intercropping, wilt, charcoal-root and monetary advantage index.

\section{Introduction}

Sesame"Sesamum indicum L." Protein (18-20 percent) and calcium, phosphorus, potassium and vitamin $\mathrm{E}$ are one of the best sources of oil (46-52 percent). A high index of sesamin, sesamol and sesamolin antioxidants and monounsaturated and polyunsaturated fatty acids are found in its high quality and staple oil (Rangkadilok et al., 2010).

Guar (Cyamopsis tetragonoloba L.) is a summer legume crop that is annual. It is dry-tolerant and grows as a vegetable for human consumption, as a feed for animals, as a green manure for soil and as a grain. Its seed is a rich source of farm-industry for the production of galactomannin, which is used in the food processing, paper making and textile printing and pharmaceutical and cosmetic industries. It is a fastgrowing, short-lived crop; thus, it suits nicely in most of the prevailing season (Alexander et al., 1988).

Sesame is infected with many soil fungi that affect the quality of the seed and the quantity of total seed production, causing damping-off of the fungus and root rot, rot-wilt diseases of charcoal. Fungi that cause certain diseases i.e. Rhizoctonia solani, Fusarium spp., Inducing significant damage and high reduction in sesame yield, Macrophomina phaseolina, Sclerotium rolfsii and Fusarium oxysporum (Morsy, 2005; El-Shennawy et al., 2010) Charcoal rot-wil is responsible for Macrophomina phaseolina and Fusarium oxysporum

are caused charcoal rot-wilt disease complex and are known to be the most harmful sesame crop fungus in all the lands of Egypt as well as the world (Khalifa, 2003, and Shabana et al., 2014). In addition to affecting the yield and causing quantitative and qualitative losses, the importance of such fungus lies in increasing soil infestation with causal pathogens. Therefore, growing sesame in this soil is not suitable for the occurrence of death of plants or reduction in yield, and seed oil content. In contrast, fungicides are extremely harmful to health and environmental contamination, so researchers plan to use induced resistance, biological control, and crop rotation as the environmentally friendly alternative methods for disease control (Khalifa, 1997, El-Fiki et al., 2004, Khalifa et al., 2007, El-Shennawy et al., 2010 and Khalifa et al., 2011).

The researchers found that there are factors that influence the spread of sesame diseases in and from various lands as resistance cultivars, sown depth, sown dates, crop rotation and intercropping; reports have been made to affect the spread of the disease in sesame fields (Khalifa, 1997, El-Garhy, 2000, and Shabana et al., 2014).

Intercropping is practically suited to develop agricultural production via increasing crop production from the available agriculture area without disturbing 
crop structure through the growing of a secondary crop combined with the main crop on the same field using some production factors of the main crop, like soil, water, fertilizer and tillage operation with high efficiency. It is not easy to bring more land into cultivation or increase crop productivity at the available area with the population increase so, intercropping has been recognized as a potential pattern for improving production in developing countries. The intercropping pattern is the best according to land utilization from land equivalent ratio (LER) and relative crowding coefficient (RCC). Sesame had higher rebuts of aggressively (dominant), while forage crops had the lowest values for aggressively (dominated) (Mokadem and ElKaramaity 1993; Abo-Kerisha et al., 2008; Mahdy and El-Said, 2015).

The objective of this investigation was to study the effect of intercropping guar with sesame under three plant densities 100, 67 and $50 \%$ from the sole as secondary crops and cut once or twice of guar on yield, yield components and reduce the incidence of Charcoal-rot and wilt disease complex under Middle Egypt conditions.

\section{Materials and Methods}

\section{I- Field experiment:}

A field trial was conducted at Mallawi Agricultural Research Station, Minia Governorate, ARC, during two successive summer seasons of 2016and 2017in a high naturally infested field with charcoal rot and wilt pathogens to study the effect of intercropping guar (Cluster bean) Cyamposis tetragonoloba cv. Local variety as secondary with crop sesame (Sesamum indicum L.) cv. Shandaweel-3 as the main crop on yield and yield components, competitive relationships and the economic return. The preceding crop was faba bean for both crops.

These experiments were laid out in a split-plot arrangement in Randomized Complete Blocks Design with three replicates. The main plots were devoted to the intercropping fodder systems, whereas the subplots were devoted to the number of cutting guar.

A1- $100 \%$ sesame $+100 \%$ guar (growing guar on the other side of all sesame ridges at $30 \mathrm{~cm}$ between the hills).

A2- $100 \%$ sesame $+67 \%$ guar (growing guar on the other side of all sesame ridges at $45 \mathrm{~cm}$ between the hills).

A3- $100 \%$ sesame $+50 \%$ guar (growing guar on the other side of all sesame ridges at $60 \mathrm{~cm}$ between the hills).

Sesame seeds in all intercropping patterns or pure stand were grown one side of all ridges in one plant/hill $10 \mathrm{~cm}$. apart.

The subplots were occupied the number of cutting of guar:

B1- One cut of guar after 60 days of sowing.
B2- Two cuts of guar the first after 60 days of sowing and the second after 45 days from the first one.

Solid fodder crops legume were three cutting after 60 days of seeding, the second after 45 days of the first and the three after 35 days of the second length of the growing season.

Solid plots of sesame and fodder crop legume guar were also included in each replication for comparison and determination of the competitive relationships and to calculate the yield advantage of crops, total income and net return /fad. Plants were sowing on May $25^{\text {th }}$ and $28^{\text {th }}$ in 2016 and 2017 seasons, respectively. The area of each plot was $10.5 \mathrm{~m}^{2}(3.5 \mathrm{~m}$. width and $3 \mathrm{~m}$. length).The plot consisted of 5 ridges spaced $70 \mathrm{~cm}$ apart.

Normal cultural practices were applied for each crop under study either in the pure stand or in intercropping as recommended for the region. However, nitrogen fertilizer was applied as ammonium nitrate $(33.5 \% \mathrm{~N})$ at a rate $100 \mathrm{~kg} \mathrm{~N}$ fad, in three equal doses Jest before the first, second and third irrigation of sesame. Calcium superphosphate $\left(15 \% \mathrm{P}_{2} \mathrm{O}_{5}\right)$ at a rate of $150 \mathrm{~kg} / \mathrm{fad}$. and potassium sulfate $\left(48 \% \mathrm{~K}_{2} \mathrm{O}\right)$ at rate of $50 \mathrm{~kg} /$ fad were applied during soil preparation. All other agricultural practices for sesame and legume fodder crop guar production were carried out as recommended. The fertilizers N, P and $\mathrm{K}$ for sole planting of both crops (main and secondary) were applied as recommended.

The different agronomic parameters were recorded of sesame and guar crops at harvesting as follows:

\section{1- Sesame crop:}

Plant height was measured as the average of ten plants at harvest after 110 days from sowing. Height of the first capsule $(\mathrm{cm})$, length of fruiting zone $(\mathrm{cm})$, number of capsules / plant, seed yield / plant (g), seed index $(\mathrm{g})$ and seed yield $\mathrm{ardab} / \mathrm{fad}(\mathrm{ardab}=120 \mathrm{~kg})$.

\section{2-Legume fodder crop guar:}

Plant height $(\mathrm{cm})$, fresh weight/plant $(\mathrm{g})$, dry weight/plant (g), total yield/fad (ton), total dry yield/fad(ton).Total fresh and dry yield/fad.(ton) were calculated for all plots and \% leaves to stems.

Competitive relationships and yield advantages: Land Equivalent Ratio (LER), Land Equivalent Coefficient (LEC), Competitive ratio (CR), Aggressivity (Agg) and Monetary advantage index (MAI) were respectively calcualuted according to (Willey 1979), (Adetiloye et al., 1983), Willey and Rao (1980), Mc-Gilichrist (1965) and Willey (1979).

Total income: It was calculated due to Agriculture Statistics (2016 and 2017) as follows: L.E 2700 for ardab of sesame; LE 300 for ton of guar as green fodder.

Net return: It was calculated by subtract total income of maize pure stand from the total income of each intercrop. 


\section{II-Greenhouse experiment:}

Fungal inoculate of Macrophomin a phaseolina and/or Fusarium oxysporum the causal pathogens of charcoal- rot and wilt diseases, respectively were prepared using sorghum-coarse sand- water $(2: 1: 2$ $\mathrm{v} / \mathrm{v})$ medium. The ingredients were mixed, bottled and autoclaved for two hours at 1.5 air pressure. The autoclaved media in glass bottles was inoculated separately using agar discs obtained from the periphery of 5- day old colony of each of the tested fungi and incubated at $26{ }^{\circ} \mathrm{C}$ for two weeks and were then used for soil infestation. The inoculate of $M$ phaseolina and $F$. oxysporum were used singly or in combination at the rate of total $2 \%$ by weight, to infest sterilized potted soil, mixed thoroughly with the soil, then watered and left for one week before sowing.

Sesame seeds, cv. Shandaweel-3 as main crop with guar (Cluster bean) Cyamposis tetragonoloba cv. Local variety as secondary crops were sown in $50 \mathrm{~cm}$ diameter pots (20 seeds of sesame against 0, 20, 13 and/or 10 of guar seeds, respectively) were distributed inside each pot/treatment to perform the following four intercropping patterns i.e., sole sesame, $100 \%$ sesame $+100 \%$ guar, $100 \%$ sesame $+65 \%$ guar and $100 \%$ sesame $+50 \%$ guar, respectively. Four pots were used as replicates.

Disease assessment was measured as percentages of pre- and post-emergence damping-off after 15 and 45 days from sowing, respectively. Percentages of diseased plants infected with charcoal-rot and/or wilt were estimated according to a specific disease symptom and recorded after 90 days from sowing. The number of uninfected plants was calculated by subtracting the whole plants from the infected and determining the infection before and after its appearance. The estimate of the disease was calculated at all stages of growth based on the number of seeds that were planted in each pot that was planted in it.

\section{Statistical analysis:}

All data in each season were statistically analyzed using analysis of variance (ANOVA) with the Statistical Analysis System MSTAT-C Statistical Packing Freed 1991. Probabilities equal to or less than 0.05 were considered significant. If ANOVA indicated differences between treatment means LSD test was performed according to Steel and Torrie 1980.

\section{Results and Discussion \\ I- Field experiments: \\ 1-Sesame: \\ A1-Effect of intercropping legume fodder crop guar with sesame:}

Intercropping guar at different plant densities on sesame had affected significantly on all characters understudy in the two seasons, except the height of the first capsule $(\mathrm{cm})$ in the two seasons and seed index in the first season and plant height in the second season (Table1). The higher values of all previous characters were recorded when intercropping guar at a rate of 50 $\%$ except the height of the first capsule recorded the lowest values. The number of capsules/plant of solid planting exceeded significantly than those three different intercropping patterns in both seasons. Seed yield / fad with sowing 50\% guar with sesame were increased by $48.44,30.37 \%$ and $39.88,22.81 \%$ than sowing $100 \%$ and $67 \%$ guar with sesame in the two seasons, respectively. Pure or sole planting of sesame scored the highest values of seed yield, were increased by $50.17,31.78,1.11 \%$ and $54.07,25.27,10.15 \%$ in the first and second seasons, respectively comparison intercropping guar on sesame. Intercropping crop guar can be used for the protection of sesame damping-off and root rot disease and this method can contribute to minimizing the risk and hazard of toxic fungicides. These results are in agreement with (Montaser and Kamal 2012; Gebregergis and Amare 2019).

The seed yield /fed of sesame for sowing $50 \%$ guar seeds between ridges was higher than sowing $100 \%$ and $67 \%$ guar which might be due to the increase in, number capsules and seeds yield /plant, seed index $(\mathrm{g})$. Low availability of light for a component crop in the mixtures reduced the photosynthetic rate and growth rate and competition between sesame and guar plants finally leading to a drastic reduction in grain yields of component crops. Yield components of sesame i.e. number of capsules/plant, seed index and seed yield /plant were superior when planted at $50 \%$ density followed by those planted at $67 \%$ density. It is clear that yield component traits were decreased consistently and regularly with increasing guar density. The lowest plant density $50 \%$ guar recorded the highest seed yield / fad. In research on intercropping of guar and sesame, different cropping arrangements had a significant effect on the biological yields of both crops. These results are in agreement with Abdel - Galil and Abdel - Chany (2014), Puste et al. (2014) and Oyeogbe et al. (2015). 
Table1. Yield traits of intercropped sesame with guar as affected by guar cutting number in 2016 and 2017 seasons.

\begin{tabular}{|c|c|c|c|c|c|c|c|c|c|c|c|c|c|c|c|}
\hline \multirow[t]{2}{*}{$\begin{array}{c}\text { Intercro } \\
\text { pping } \\
\text { Pattern }\end{array}$} & \multirow[t]{2}{*}{$\begin{array}{c}\text { No } \\
\text { of } \\
\text { Cutt } \\
\text { ing }\end{array}$} & \multicolumn{2}{|c|}{$\begin{array}{c}\text { Plant } \\
\text { height } \mathrm{cm} \text {. }\end{array}$} & \multicolumn{2}{|c|}{$\begin{array}{c}\text { Height of } \\
\text { the first } \\
\text { Capsule } \\
\text { cm, }\end{array}$} & \multicolumn{2}{|c|}{$\begin{array}{l}\text { Length of } \\
\text { fruiting } \\
\text { zone } \mathrm{cm} .\end{array}$} & \multicolumn{2}{|c|}{$\begin{array}{c}\text { Number of } \\
\text { Capsules / } \\
\text { plant }\end{array}$} & \multicolumn{2}{|c|}{$\begin{array}{c}\text { Seed } \\
\text { Yield } \\
\text { /plant g. }\end{array}$} & \multicolumn{2}{|c|}{$\begin{array}{l}\text { Seed } \\
\text { index }\end{array}$} & \multicolumn{2}{|c|}{$\begin{array}{c}\text { Seed } \\
\text { yield } \\
\text { ardab/fa } \\
\text { d }\end{array}$} \\
\hline & & 201 & 201 & 20 & 20 & 201 & 201 & 201 & 201 & 20 & 20 & 20 & 20 & 20 & 20 \\
\hline \multirow{3}{*}{$\begin{array}{c}\text { Sesame+ } \\
100 \% \\
\text { guar }\end{array}$} & & 6 & 7 & 16 & 17 & 6 & 7 & 6 & 7 & 16 & 17 & 16 & 17 & 16 & 17 \\
\hline & 1cut & $\begin{array}{c}152 . \\
89\end{array}$ & $\begin{array}{c}157 . \\
02\end{array}$ & $\begin{array}{r}60 . \\
00\end{array}$ & $\begin{array}{r}66 . \\
67\end{array}$ & $\begin{array}{c}92.6 \\
7\end{array}$ & $\begin{array}{c}87.6 \\
7\end{array}$ & 67.5 & $\begin{array}{c}61.6 \\
7\end{array}$ & $\begin{array}{l}11 . \\
16\end{array}$ & $\begin{array}{l}11 . \\
30\end{array}$ & $\begin{array}{c}3.2 \\
8\end{array}$ & $\begin{array}{c}3.7 \\
7\end{array}$ & $\begin{array}{c}3.5 \\
0\end{array}$ & $\begin{array}{c}3.3 \\
9\end{array}$ \\
\hline & 2cut & $\begin{array}{c}148 . \\
08\end{array}$ & $\begin{array}{c}154 . \\
89\end{array}$ & $\begin{array}{l}59 . \\
00\end{array}$ & $\begin{array}{r}65 . \\
67\end{array}$ & $\begin{array}{c}89.0 \\
6\end{array}$ & $\begin{array}{c}89.2 \\
2\end{array}$ & $\begin{array}{c}58.8 \\
0\end{array}$ & $\begin{array}{c}54.5 \\
7\end{array}$ & $\begin{array}{l}13 . \\
31\end{array}$ & $\begin{array}{l}13 . \\
81\end{array}$ & $\begin{array}{c}2.9 \\
8\end{array}$ & $\begin{array}{c}3.3 \\
8\end{array}$ & $\begin{array}{c}2.5 \\
2\end{array}$ & $\begin{array}{c}3.2 \\
2\end{array}$ \\
\hline \multicolumn{2}{|c|}{ Mean } & $\begin{array}{c}150 . \\
48\end{array}$ & $\begin{array}{c}155 . \\
96\end{array}$ & $\begin{array}{l}59 . \\
50\end{array}$ & $\begin{array}{l}66 . \\
17\end{array}$ & $\begin{array}{c}90.8 \\
6\end{array}$ & $\begin{array}{c}88.4 \\
5\end{array}$ & $\begin{array}{c}63.1 \\
5\end{array}$ & $\begin{array}{c}58.1 \\
2\end{array}$ & $\begin{array}{l}12 . \\
24\end{array}$ & $\begin{array}{l}12 . \\
55\end{array}$ & $\begin{array}{c}3.1 \\
3\end{array}$ & $\begin{array}{c}3.5 \\
8\end{array}$ & $\begin{array}{c}3.0 \\
1\end{array}$ & $\begin{array}{c}3.3 \\
1\end{array}$ \\
\hline \multirow{2}{*}{$\begin{array}{l}\text { Sesame+ } \\
67 \% \\
\text { guar }\end{array}$} & 1cut & $\begin{array}{c}165 . \\
05\end{array}$ & $\begin{array}{c}166 . \\
83\end{array}$ & $\begin{array}{c}56 . \\
00\end{array}$ & $\begin{array}{l}55 . \\
28\end{array}$ & $\begin{array}{c}109 . \\
00\end{array}$ & $\begin{array}{c}111 . \\
50\end{array}$ & $\begin{array}{c}81.6 \\
3\end{array}$ & $\begin{array}{c}86.6 \\
8\end{array}$ & $\begin{array}{l}12 . \\
49\end{array}$ & $\begin{array}{l}14 . \\
73\end{array}$ & $\begin{array}{c}3.3 \\
5\end{array}$ & $\begin{array}{c}4.1 \\
9\end{array}$ & $\begin{array}{c}3.9 \\
0\end{array}$ & $\begin{array}{c}3.9 \\
9\end{array}$ \\
\hline & 2cut & $\begin{array}{c}157 . \\
11\end{array}$ & $\begin{array}{c}167 . \\
00\end{array}$ & $\begin{array}{r}53 . \\
67\end{array}$ & $\begin{array}{c}53 . \\
50\end{array}$ & $\begin{array}{c}101 . \\
67\end{array}$ & $\begin{array}{c}107 . \\
33\end{array}$ & $\begin{array}{c}70.5 \\
4\end{array}$ & $\begin{array}{c}80.0 \\
8\end{array}$ & $\begin{array}{l}14 . \\
34\end{array}$ & $\begin{array}{l}15 . \\
39\end{array}$ & $\begin{array}{c}3.0 \\
7\end{array}$ & $\begin{array}{c}3.4 \\
6\end{array}$ & $\begin{array}{c}2.9 \\
6\end{array}$ & $\begin{array}{c}3.5 \\
4\end{array}$ \\
\hline \multicolumn{2}{|c|}{ Mean } & $\begin{array}{c}161 . \\
08\end{array}$ & $\begin{array}{c}166 . \\
92\end{array}$ & $\begin{array}{l}54 . \\
83\end{array}$ & $\begin{array}{l}54 . \\
39\end{array}$ & $\begin{array}{c}105 . \\
33\end{array}$ & $\begin{array}{c}109 . \\
42\end{array}$ & $\begin{array}{c}76.0 \\
9\end{array}$ & $\begin{array}{c}83.3 \\
7\end{array}$ & $\begin{array}{l}13 . \\
42\end{array}$ & $\begin{array}{l}15 . \\
07\end{array}$ & $\begin{array}{c}3.2 \\
1\end{array}$ & $\begin{array}{c}3.8 \\
3\end{array}$ & $\begin{array}{c}3.4 \\
3\end{array}$ & $\begin{array}{c}3.7 \\
7\end{array}$ \\
\hline $\begin{array}{l}\text { Sesame+ } \\
\mathbf{5 0 \%} \\
\text { guar }\end{array}$ & 2cut & $\begin{array}{c}178 . \\
44 \\
168 . \\
89\end{array}$ & $\begin{array}{c}180 . \\
00 \\
166 . \\
00\end{array}$ & $\begin{array}{r}52 . \\
33 \\
50 . \\
67\end{array}$ & $\begin{array}{r}48 . \\
67 \\
49 . \\
67\end{array}$ & $\begin{array}{c}126 . \\
00 \\
118 . \\
00\end{array}$ & $\begin{array}{c}134 . \\
00 \\
123 . \\
67\end{array}$ & $\begin{array}{c}105 . \\
67 \\
94.0 \\
7\end{array}$ & $\begin{array}{c}110 . \\
00 \\
104 . \\
00\end{array}$ & $\begin{array}{l}13 . \\
96 \\
14 . \\
99\end{array}$ & $\begin{array}{l}15 . \\
09 \\
15 . \\
00\end{array}$ & $\begin{array}{c}3.7 \\
2 \\
3.4 \\
3\end{array}$ & $\begin{array}{c}4.3 \\
9 \\
3.9 \\
9\end{array}$ & $\begin{array}{c}5.1 \\
4 \\
3.7 \\
9\end{array}$ & $\begin{array}{c}5.1 \\
6 \\
4.0 \\
9\end{array}$ \\
\hline Mean & & $\begin{array}{c}173 . \\
67\end{array}$ & $\begin{array}{c}173 . \\
00\end{array}$ & $\begin{array}{l}51 . \\
50\end{array}$ & $\begin{array}{l}49 . \\
17\end{array}$ & $\begin{array}{c}122 . \\
00\end{array}$ & $\begin{array}{c}128 . \\
83\end{array}$ & $\begin{array}{c}99.8 \\
7\end{array}$ & $\begin{array}{c}107 . \\
00\end{array}$ & $\begin{array}{r}14 . \\
48\end{array}$ & $\begin{array}{l}15 . \\
05\end{array}$ & $\begin{array}{c}3.5 \\
8\end{array}$ & $\begin{array}{c}4.1 \\
9\end{array}$ & $\begin{array}{c}4.4 \\
7\end{array}$ & $\begin{array}{c}4.6 \\
3\end{array}$ \\
\hline \multirow{3}{*}{$\begin{array}{l}\text { Average } \\
\text { cutting }\end{array}$} & 1cut & $\begin{array}{c}156 . \\
46\end{array}$ & $\begin{array}{c}167 . \\
95\end{array}$ & $\begin{array}{r}56 . \\
11\end{array}$ & $\begin{array}{l}56 . \\
87\end{array}$ & $\begin{array}{c}129 . \\
00\end{array}$ & $\begin{array}{c}111 . \\
06\end{array}$ & $\begin{array}{c}84.9 \\
3\end{array}$ & $\begin{array}{c}86.1 \\
2\end{array}$ & $\begin{array}{l}12 . \\
54\end{array}$ & $\begin{array}{l}13 . \\
70\end{array}$ & $\begin{array}{c}3.4 \\
5\end{array}$ & $\begin{array}{c}4.1 \\
2\end{array}$ & $\begin{array}{c}4.1 \\
8\end{array}$ & $\begin{array}{c}4.1 \\
8\end{array}$ \\
\hline & 2cut & $\begin{array}{c}158 . \\
03\end{array}$ & $\begin{array}{c}162 . \\
63\end{array}$ & $\begin{array}{c}54 . \\
50\end{array}$ & $\begin{array}{l}56 . \\
28\end{array}$ & $\begin{array}{c}121 . \\
00\end{array}$ & $\begin{array}{c}106 . \\
74\end{array}$ & $\begin{array}{c}74.4 \\
7\end{array}$ & $\begin{array}{c}79.5 \\
4\end{array}$ & $\begin{array}{l}14 . \\
21\end{array}$ & $\begin{array}{l}14 . \\
73\end{array}$ & $\begin{array}{c}3.1 \\
6\end{array}$ & $\begin{array}{c}3.6 \\
1\end{array}$ & $\begin{array}{c}3.0 \\
9\end{array}$ & $\begin{array}{c}3.6 \\
2\end{array}$ \\
\hline & $\mathbf{A}$ & 6.76 & N.s & N.s & N.s & $\begin{array}{c}16.3 \\
3\end{array}$ & $\begin{array}{c}20.0 \\
3\end{array}$ & $\begin{array}{c}13.6 \\
9\end{array}$ & $\begin{array}{c}7.42 \\
2\end{array}$ & $\begin{array}{c}1.5 \\
9\end{array}$ & $\begin{array}{c}0.9 \\
2\end{array}$ & $\begin{array}{c}\mathbf{N} . \\
\mathbf{S}\end{array}$ & $\begin{array}{c}0.5 \\
4\end{array}$ & $\begin{array}{c}0.7 \\
7\end{array}$ & $\begin{array}{c}0.5 \\
8\end{array}$ \\
\hline \multirow{2}{*}{ LSD5\% } & B & 5.96 & N.s & N.s & N.s & 4.65 & N.s & 9.09 & 4.64 & $\begin{array}{c}1.2 \\
9\end{array}$ & $\begin{array}{c}1.2 \\
2\end{array}$ & $\begin{array}{c}0.2 \\
9\end{array}$ & $\begin{array}{c}0.4 \\
8\end{array}$ & $\begin{array}{c}0.8 \\
7\end{array}$ & $\begin{array}{c}0.4 \\
1\end{array}$ \\
\hline & $\mathbf{A B}$ & N.s & N.s & N.s & N.s & N.S & N.S & N.s & N.s & N.S & N.s & $\begin{array}{c}\mathbf{N} . \\
\mathbf{S}\end{array}$ & $\begin{array}{c}\mathbf{N} . \\
\mathbf{S}\end{array}$ & $\begin{array}{c}\mathbf{N} . \\
\mathbf{S}\end{array}$ & N.s \\
\hline \multicolumn{2}{|c|}{ Sole sesame } & $\begin{array}{c}165 . \\
25\end{array}$ & $\begin{array}{c}177 . \\
67\end{array}$ & $\begin{array}{c}56 . \\
58\end{array}$ & $\begin{array}{r}66 . \\
67\end{array}$ & $\begin{array}{c}108 . \\
67\end{array}$ & $\begin{array}{c}111 . \\
00\end{array}$ & $\begin{array}{l}110 . \\
5\end{array}$ & $\begin{array}{c}106 . \\
67\end{array}$ & $\begin{array}{l}13 . \\
84\end{array}$ & $\begin{array}{l}14 . \\
27\end{array}$ & $\begin{array}{c}3.0 \\
5\end{array}$ & $\begin{array}{c}3.5 \\
4\end{array}$ & $\begin{array}{c}4.5 \\
2\end{array}$ & $\begin{array}{l}5.1 \\
02\end{array}$ \\
\hline
\end{tabular}

Amanullah, (2017) found that yield components, groundnut had significantly fewer pods plant ${ }^{-1}$, kernel weight, grain yield, and weight of 100 seed (g) when intercropped sorghum with millet as compared with the other members of its own family mungbean with guar and sesame. This is due to the greater utilization of lighting and nutrients with intercropping systems compared to single cultivation for each of them, in addition to benefiting from the presence of leguminous crops.

B1- Effect of guar cutting number on sesame:

The result presented in (Table1) reveal that the no of cutting of guar had significant effect on all characters under study of two seasons, except plant height, Length of fruiting zone in the second seasons and height of the first capsule in the two seasons. The highest sesame seed yield was recorded when cutting guar once of $4.18 \mathrm{ardab} / \mathrm{fad}$ in the two seasons, the lowest sesame seed yield was recorded when cutting guar twice i.e. 3.09 and $3.62 \mathrm{ardab} / \mathrm{fad}$ in the 1 st and $2^{\text {nd }}$ seasons, respectively.

\section{2- Legume forage crop guar:}

A2-Effect of intercropping guar plant density:

Data presented in Table (2) revealed that the effect of three rates of guar plant densities in intercropping with sesame $(100 \%, 67 \%$ and $50 \%)$, did not show significant effect on all studied characters of guar, except total fresh yield /fad and total dry yield/fad of guar which were significantly influenced by intercropped guar at different plant densities with sesame. Concerning total fresh and dry yield/fad. Intercropping patterns significantly decreased green forage yield/fad, compared to sole guar sowing in both seasons Table 2.The total fresh weight/ plant of guar at 100,67 and $50 \%$ as compared to sole. 
Table 2. Yield traits of intercropped guar with sesame as affected by guar cutting no. in 2016 and 2017 seasons.

\begin{tabular}{|c|c|c|c|c|c|c|c|c|c|c|c|c|c|}
\hline \multirow[t]{2}{*}{$\begin{array}{c}\text { Intercropping } \\
\text { Pattern }\end{array}$} & \multirow[t]{2}{*}{$\begin{array}{c}\begin{array}{c}\text { No of } \\
\text { cutting }\end{array} \\
\end{array}$} & \multicolumn{2}{|c|}{ Plant height $(\mathbf{c m})$} & \multicolumn{2}{|c|}{$\begin{array}{l}\text { Fresh weight / } \\
\text { Plant (g) }\end{array}$} & \multicolumn{2}{|c|}{$\begin{array}{l}\text { Dry weight / } \\
\text { Plant (g) }\end{array}$} & \multicolumn{2}{|c|}{$\begin{array}{c}\text { Total fresh } \\
\text { Yield/ fed (ton) }\end{array}$} & \multicolumn{2}{|c|}{$\begin{array}{l}\text { Total dry } \\
\text { yield } \\
\text { / fed (ton) }\end{array}$} & \multicolumn{2}{|c|}{$\%$ leaves to stem } \\
\hline & & 2016 & 2017 & 2016 & 2017 & 2016 & 2017 & 2016 & 2017 & 2016 & 2017 & 2016 & 2017 \\
\hline Sesame+ & 1cut & 91.67 & 94.91 & 89.20 & 92.41 & 11.73 & 12.42 & 5.61 & 6.32 & 0.73 & 0.85 & 51.32 & 53.55 \\
\hline $100 \%$ guar & 2cut & 86.10 & 89.35 & 65.67 & 68.88 & 8.91 & 9.83 & 6.21 & 6.88 & 0.85 & 1.06 & 23.05 & 25.28 \\
\hline Mean & & 88.8 & 92.13 & $\mathbf{7 7 . 4 3}$ & 80.64 & 10.31 & 11.13 & 5.91 & 6.60 & 0.79 & 0.96 & 37.18 & 39.42 \\
\hline Sesame+ & 1cut & 96.33 & 101.56 & 92.33 & 96.34 & 12.40 & 13.29 & 3.88 & 3.83 & 0.52 & 0.52 & 57.63 & 60.85 \\
\hline $67 \%$ guar & 2cut & 89.73 & 91.96 & 71.67 & 75.68 & 9.52 & 10.41 & 4.38 & 4.94 & 0.58 & 0.68 & 32.64 & 35.95 \\
\hline Mean & & 93.03 & 96.76 & 82.00 & 86.01 & 10.96 & 11.85 & 4.13 & 4.39 & 0.55 & 0.60 & 45.14 & 48.40 \\
\hline Sesame+ & 1cut & 100.33 & 104.54 & 107.30 & 112.51 & 15.93 & 17.22 & 3.28 & 4.12 & 0.49 & 0.63 & 68.05 & 71.71 \\
\hline $50 \%$ guar & 2cut & 97.00 & 96.11 & $\mathbf{7 7 . 7 8}$ & 83.00 & 12.07 & 13.36 & 4.29 & 4.78 & 0.68 & 0.79 & 37.22 & 40.87 \\
\hline Mean & & 98.67 & 100.33 & 92.54 & 97.76 & 14.00 & 15.29 & 3.79 & 4.45 & 0.59 & 0.71 & 52.64 & 56.29 \\
\hline \multirow{3}{*}{$\begin{array}{l}\text { Average } \\
\text { cutting }\end{array}$} & 1cut & 96.11 & 100.34 & 96.28 & 100.42 & 13.36 & 14.31 & 4.26 & 4.76 & 0.58 & 0.66 & $\mathbf{5 9 . 0 0}$ & 62.04 \\
\hline & 2 cut & 90.94 & 92.47 & 71.71 & 75.95 & 10.16 & 11.20 & 4.96 & 5.53 & 0.71 & 0.84 & 30.97 & 34.03 \\
\hline & A & N.s & N.s & N.s & $\mathbf{N}$ & N.s & N.s & 0.45 & 1.25 & 0.18 & 0.27 & N.s & N.s \\
\hline \multirow{2}{*}{ LSD5\% } & B & N.s & N.s & 5.91 & 5.91 & 2.20 & 2.19 & N.s & N.s & 0.12 & 0.13 & 4.72 & 4.72 \\
\hline & AB & N.s & N.s & N.s & N.s & N.s & N.s & N.s & N.s & N.s & N.s & N.s & N.s \\
\hline Sole gi & & 92.56 & $\mathbf{9 7 . 4 5}$ & 89.12 & 101.15 & 14.58 & 16.16 & 12.21 & 13.71 & 2.00 & 2.19 & 77.95 & 81.52 \\
\hline
\end{tabular}

crop were $44.31,33.82$ and $39.23 \%$, respectively in the $1^{\text {st }}$ season, $48.14,32.62$ and $32.45 \%$, respectively in the $2^{\text {nd }}$ season. The highest values of total fresh yield/ fad (ton) and total dry yield/ fad (ton) of guar with sesame when intercropped at dentistry of $100 \%$ were 5.91, 0.79 and $6.60,0.46$ ton /fad in the $1^{\text {st }}$ and 2 nd seasons, respectively. This is because that the growth of sesame plants led to weak growth of intercropped legume forage crop guar which led to cutting once and the solo crop was cutting twice until the harvest of sesame. \% leaves / stem recorded highest values when intercropping $50 \%$ guar with sesame and one cut of crop guar i.e., $67.53 \& 69.05 \%$ in the $1^{\text {st }}$ and $2^{\text {nd }}$ seasons, respectively. Its importance is due to the extent to which animals love the leaves more than the stems and increase the food use of the green leaves in the green fodder crop. These results are in agreement with those obtained by Montaser and Kamal 2012; Mahdy and El-Said 2015. Amanullah, (2017).

\section{B2- Effect of guar cutting number:}

Results revealed that yield and component of guar were significantly affected by a number of cutting on (fresh weight / plant (g), dry weight / plant (g), dry yield/ fad (ton) and \% leaves to stems of guar in the two seasons).Concerning to total fresh and dry yield /fad (Table 2), the results show that the highest values of total fresh and dry yield/fad were obtained with two cuts of legume forage crop guar of 4.96,0.71 and $5.53,0.84 \mathrm{ton} / \mathrm{fad}$ in the 1 st and 2 nd seasons, respectively. These obtained results are in accordance with those obtained by Montaser and Kamal; Mahdy and El-Said 2015.

\section{c- Interaction effect:}

All interactions between intercropping legume forage crop guar and its number of cutting did not show a significant effect for all studied traits in the first and second seasons.

\section{D-Competitive relationships:-}

\section{- land equivalent ratio (LER)}

Results in Table $3 \& 4$ represent competitive relationships of intercropping sesame with guar in the 2016 and 2017 seasons. The values of land equivalent ratio for intercropping treatments were greater than monoculture (it was the same (1.0) for all pure stands of main crop and intercrops). Intercropping $50 \%$ guar with sesame and one cut of legume crop guar recorded the highest values for (LER) which were $1.41 \& 1.31$ in the $1^{\text {st }}$ and $2^{\text {nd }}$ seasons, respectively. Intercropping $67 \%$ guar on sesame with two cuts of guar recorded the lowest values for (LER) which were $1.01 \& 1.05$ in the $1{ }^{\text {st }}$ and $2^{\text {nd }}$ seasons, respectively. Similar results were obtained by Mokadem and El-Karamaity 1993; AboKerisha et al.,2008; Mahdy and El-Said 2015, Who found that LER values were greater with the intercropping system than a sole crop of them.

Abdel - Galil and Abdel - Chany (2014) studied Land equivalent ratio (LER) of the cropping system and revealed that intercropping was more economical than a sole growing of groundnut even though intercropping had reduced pod yield of groundnut in most of the case tested here. The maximum monetary advantage was also recorded for the groundnut/maize intercropping system although monoculture production of cereal fodder or groundnut yielded higher than in the intercropped culture (Gosh, 2004). ). Due to the leguminous nature of both crops, the greater LER of groundnut + guar intercropping is not surprising, but the intercropping of groundnut + sesame with the next largest LER is important. 
Table 3. Calculated data of competitive relationship and yield advantage for intercropping of guar with sesame and no of cutting on guar in 2016 season

\begin{tabular}{|c|c|c|c|c|c|c|c|c|c|c|}
\hline \multirow{3}{*}{$\begin{array}{l}\text { Intercropping } \\
\text { patterns } \\
\text { (A) }\end{array}$} & \multirow{3}{*}{$\begin{array}{l}\text { No of } \\
\text { cutting } \\
\text { (B) }\end{array}$} & \multicolumn{3}{|c|}{ Land equivalent ratio } & \multirow{3}{*}{$\mathrm{LC}$} & \multicolumn{3}{|c|}{ Competitive ratio (CR) } & \multirow{2}{*}{\multicolumn{2}{|c|}{$\begin{array}{c}\text { Aggressivity } \\
\text { (Agg) }\end{array}$}} \\
\hline & & LS & $+\mathrm{LG}=$ & E R & & $\mathrm{CR}$ & CRS & $R G$ & & \\
\hline & & $\mathrm{LS}$ & $\mathrm{LG}$ & LER & & CRS & CRG & $\mathrm{CR}$ & AS & $\mathrm{AG}$ \\
\hline \multirow{2}{*}{$\begin{array}{l}100 \% \text { sesame } \\
+100 \% \text { guar }\end{array}$} & 1 cut & 0.77 & 0.46 & 1.23 & 0.36 & 1.69 & 0.59 & 2.28 & +0.63 & -0.63 \\
\hline & 2 cut & 0.56 & 0.51 & 1.07 & 0.28 & 1.13 & 0.91 & 2.04 & +0.10 & -0.10 \\
\hline \multirow{3}{*}{$\begin{array}{l}\text { Mean } \\
\qquad \begin{array}{l}100 \% \text { sesame } \\
+67 \% \text { guar }\end{array}\end{array}$} & & 0.67 & 0.48 & 1.15 & 0.32 & 1.38 & 0.73 & 2.10 & +0.36 & -0.36 \\
\hline & $1 \mathrm{cut}$ & 0.86 & 0.32 & 1.18 & 0.27 & 1.82 & 0.55 & 2.37 & +0.65 & -0.65 \\
\hline & $2 \mathrm{cut}$ & 0.65 & 0.36 & 1.01 & 0.23 & 1.22 & 0.82 & 2.04 & +0.20 & -0.20 \\
\hline \multicolumn{2}{|l|}{ Mean } & 0.76 & 0.34 & 1.10 & 0.26 & 1.50 & 0.67 & 2.17 & +0.42 & -0.42 \\
\hline $100 \%$ sesame & 1cut & 1.14 & 0.27 & 1.41 & 0.31 & 2.07 & 0.48 & 2.55 & +0.88 & -0.88 \\
\hline$+50 \%$ guar & 2 cut & 0.84 & 0.35 & 1.19 & 0.29 & 1.17 & 0.85 & 2.02 & +0.19 & -0.19 \\
\hline \multicolumn{2}{|l|}{ Mean } & 0.99 & 0.31 & 1.30 & 0.31 & 1.56 & 0.64 & 2.20 & +0.54 & -0.54 \\
\hline
\end{tabular}

Table 4. Calculated data of competitive relationship and yield advantage for intercropping of guar with sesame and no of cutting on guar in 2017 season.

\begin{tabular}{|c|c|c|c|c|c|c|c|c|c|c|}
\hline \multirow{2}{*}{$\begin{array}{l}\text { Intercropping } \\
\text { patterns } \\
\text { (A) }\end{array}$} & \multirow{2}{*}{$\begin{array}{l}\text { No of } \\
\text { cutting }\end{array}$} & \multicolumn{3}{|c|}{ Land equivalent } & \multirow[t]{2}{*}{$\mathrm{LC}$} & \multicolumn{3}{|c|}{$\begin{array}{l}\text { Competitive ratio }(\mathrm{CR}) \\
\qquad \mathrm{CR}=\mathrm{CRS}+\mathrm{CRG}\end{array}$} & \multicolumn{2}{|c|}{$\begin{array}{l}\text { Aggressivity } \\
\text { (Agg) }\end{array}$} \\
\hline & & LS & LG & LER & & CRS & CRG & $\overline{C R}$ & AS & $\mathrm{AG}$ \\
\hline \multirow{2}{*}{$\begin{array}{l}100 \% \text { sesame } \\
+100 \% \text { guar }\end{array}$} & $1 \mathrm{cut}$ & 0.66 & 0.46 & 1.13 & 0.31 & 1.44 & 0.69 & 2.14 & +0.41 & -0.41 \\
\hline & $2 \mathrm{cut}$ & 0.63 & 0.50 & 1.13 & 0.32 & 1.26 & 0.80 & 2.05 & +0.26 & -0.26 \\
\hline \multicolumn{2}{|l|}{ Mean } & 0.65 & 0.48 & 1.13 & 0.31 & 1.35 & 0.74 & 2.09 & +0.33 & -0.33 \\
\hline \multirow{2}{*}{$\begin{array}{l}100 \% \text { sesame } \\
+67 \% \text { guar }\end{array}$} & $1 \mathrm{cut}$ & 0.78 & 0.28 & 1.06 & 0.22 & 1.88 & 0.54 & 2.41 & +0.61 & -0.61 \\
\hline & $2 \mathrm{cut}$ & 0.69 & 0.36 & 1.05 & 0.25 & 1.29 & 0.78 & 2.07 & +0.26 & -0.26 \\
\hline \multicolumn{2}{|l|}{ Mean } & 0.74 & 0.32 & 1.06 & 0.24 & 1.55 & 0.65 & 2.20 & +0.43 & -0.43 \\
\hline \multirow[t]{2}{*}{$\begin{array}{l}100 \% \text { sesame } \\
+50 \% \text { guar }\end{array}$} & 1cut & 1.01 & 0.30 & 1.31 & 0.30 & 1.65 & 0.60 & 2.25 & +0.60 & -0.60 \\
\hline & $2 \mathrm{cut}$ & 0.80 & 0.35 & 1.15 & 0.28 & 1.13 & 0.88 & 2.01 & +0.14 & -0.14 \\
\hline \multicolumn{2}{|l|}{ Mean } & 0.91 & 0.32 & 1.23 & 0.29 & 1.37 & 0.73 & 2.10 & +0.37 & -0.37 \\
\hline
\end{tabular}

-Land equivalent coefficient (LEC) is a measure of interaction concerned with the strength of the relationship.LEC is used for two -crop mixture the minimum expected productivity coefficient (PC) $25 \%$, that is, a yield advantage is obtained if the LEC value exceeded 0.25 . The effects of intercropping pattern and number of cutting and their interaction on the LEC of intercropping guar with sesame exceeded 0.25 in the intercrop combinations used in this study, except intercropping $67 \%$ guar with sesame and two cuts of guar in the $1^{\text {st }}$ season and one cut of guar in the $2^{\text {nd }}$ season. Mean LEC values varied from $0.23,0.22$ and $0.36,0.32$ in the two seasons, respectively (Table3\&4).LEC values followed a trend similar to that of LER. This is consistent with the findings of Nassef and Abd El-Gaid 2012 and Wafaa et al., 2013.

- Effect of various cropping systems on the competitive ratio $(\mathbf{C R})$ :
Data presented in Tables $3 \& 4$ reveal that the lowest values of CR were recorded for intercropping sesame with $50 \%$ guar and two cuts of guar 2.02 and 2.01 in the $1^{\text {st }}$ and $2^{\text {nd }}$ seasons, respectively. However, the highest value of $\mathrm{CR}$ was recorded for intercropping sesame with cutting guar once at densities of 50 and $70 \%$ one cut of guar $2.55,2.41$ in the $1^{\text {st }}$ and $2^{\text {nd }}$ seasons, respectively. Similar results were recorded by Mahdy and El-said2015; Wafaa $\boldsymbol{e t}$ al., 2013.

- Effect of various cropping systems on Aggressivity(Agg):

Data in Tables $3 \& 4$ show that aggressivity values of sesame were positive, whereas values of legume fodder crop guar were negative in both seasons, meaning that sesame was dominant and guar was dominated. Similar results were recorded by Mokadem and El-Karamaity 1993; AboKerisha $e t$ al.,2008; Mahdy and El-Said2015. 
Table 5. Economic analysis of intercropping pattern and no of cutting of guar in 2016 season.

\begin{tabular}{|c|c|c|c|c|c|c|c|c|c|}
\hline \multirow{3}{*}{$\begin{array}{l}\text { Intercropping } \\
\text { patterns } \\
\text { (A) }\end{array}$} & \multirow{3}{*}{$\begin{array}{c}\text { No } \\
\text { of } \\
\text { cutting }\end{array}$} & \multirow{2}{*}{\multicolumn{2}{|c|}{ Crop yield }} & \multirow{2}{*}{\multicolumn{2}{|c|}{$\begin{array}{c}\text { Total } \\
\text { Income (LE /fed) }\end{array}$}} & \multirow{3}{*}{$\begin{array}{c}\text { Total } \\
547 \text { nco } \\
\text { me } \\
(\mathrm{LE} \\
/ \text { fed) }\end{array}$} & \multirow{3}{*}{$\begin{array}{c}\text { Total } \\
\text { expenditure } \\
\text { (LE /fed) }\end{array}$} & \multirow{3}{*}{$\begin{array}{l}\text { Net } \\
\text { profit } \\
\text { (LE } \\
/ \text { fed) }\end{array}$} & \multirow{3}{*}{ MAI } \\
\hline & & & & & & & & & \\
\hline & & $\begin{array}{l}\text { Sesame } \\
\text { ( Ardb } \\
\text { /fad }\end{array}$ & $\begin{array}{l}\text { Guar } \\
\text { ton/fad }\end{array}$ & Sesame & $\begin{array}{l}\text { Guar } \\
\text { ton/fad }\end{array}$ & & & & \\
\hline \multirow{2}{*}{ A1 } & B1 & 3.5 & 5.61 & 9.450 & 1.683 & 11.133 & 6.842 & 4.291 & 3.48 \\
\hline & B2 & 2.52 & 6.21 & 6.804 & 1.863 & 8.667 & 6.962 & 1.706 & 0.77 \\
\hline Mean & & 3.01 & 5.91 & 8.127 & 1.773 & 9.900 & 6.902 & 2.998 & 2.13 \\
\hline \multirow{2}{*}{$\mathrm{A} 2$} & B1 & 3.9 & 3.88 & 10.530 & 1.164 & 11.694 & 6.694 & 5.00 & 4.15 \\
\hline & B2 & 2.96 & 4.38 & 7.992 & 1.314 & 9.306 & 6.814 & 2.492 & 1.51 \\
\hline \multicolumn{2}{|l|}{ Mean } & 3.43 & 4.13 & 9.261 & 1.239 & 10.500 & 6.754 & 3.746 & 2.83 \\
\hline \multirow{2}{*}{ A3 } & B1 & 5.14 & 3.28 & 13.878 & 0.984 & 14.862 & 6.617 & 8.245 & 7.53 \\
\hline & B2 & 3.79 & 4.29 & 10.233 & 1.287 & 11.520 & 6.737 & 4.783 & 3.94 \\
\hline \multicolumn{2}{|l|}{ Mean } & 4.47 & 3.79 & 12.069 & 1.137 & 13.206 & 6.677 & 6.529 & 5.76 \\
\hline \multicolumn{2}{|c|}{ Solid Sesame } & \multicolumn{2}{|c|}{4.52} & 12.204 & -- & 12.204 & 6.272 & 5.932 & 5.15 \\
\hline \multicolumn{2}{|c|}{ Solid Guar } & \multicolumn{2}{|c|}{12.21} & -- & 3.663 & 3.663 & 2650 & 2.013 & 1.10 \\
\hline
\end{tabular}

Table 6. Economic analysis of intercropping pattern and no of cutting of guar in 2017season.

\begin{tabular}{|c|c|c|c|c|c|c|c|c|c|}
\hline \multirow{2}{*}{$\begin{array}{l}\text { Intercropping } \\
\text { patterns } \\
\text { (A) }\end{array}$} & \multirow{2}{*}{$\begin{array}{c}\text { No of } \\
\text { cutting }\end{array}$} & \multicolumn{2}{|c|}{ Crop yield } & \multicolumn{2}{|c|}{$\begin{array}{c}\text { Total } \\
\text { Income (LE /fed) }\end{array}$} & \multirow{2}{*}{$\begin{array}{c}\text { Total } \\
\text { income } \\
\text { (LE } \\
\text { /fed) }\end{array}$} & \multirow{2}{*}{$\begin{array}{c}\text { Total } \\
\text { expenditure } \\
\text { (LE /fed) }\end{array}$} & \multirow{2}{*}{$\begin{array}{l}\text { Net } \\
\text { profit } \\
\text { (LE } \\
\text { /fed) }\end{array}$} & \multirow[b]{2}{*}{ MAI } \\
\hline & & $\begin{array}{l}\text { Sesame } \\
\text { ( Ardb } \\
\text { /fad }\end{array}$ & $\begin{array}{l}\text { Guar } \\
\text { ton/fad }\end{array}$ & Sesame & $\begin{array}{l}\text { Guar } \\
\text { ton/fad }\end{array}$ & & & & \\
\hline \multirow{2}{*}{ A1 } & B1 & 3.39 & 6.32 & 9.153 & 1.896 & 11.049 & 6.842 & 4.207 & 3.32 \\
\hline & B2 & 3.22 & 6.88 & 8.694 & 2.064 & 10.758 & 6.962 & 3.796 & 2.91 \\
\hline Mean & & 3.31 & 6.6 & 8.937 & 1.980 & 10.917 & 6.902 & 4.015 & 3.13 \\
\hline \multirow{2}{*}{ A2 } & B1 & 3.99 & 3.83 & 10.773 & 1.149 & 11.922 & 6.694 & 5.228 & 4.29 \\
\hline & B2 & 3.54 & 4.94 & 9.558 & 1.482 & 11.040 & 6.814 & 4.226 & 3.28 \\
\hline Mean & & 3.77 & 4.39 & 10.179 & 1.317 & 11.496 & 6.754 & 4.742 & 3.80 \\
\hline \multirow{2}{*}{ A3 } & B1 & 5.16 & 4.12 & 13.932 & 1.236 & 15.168 & 6.617 & 8.551 & 7.79 \\
\hline & B2 & 4.09 & 4.78 & 11.043 & 1.434 & 12.477 & 6.737 & 5.74 & 4.87 \\
\hline \multicolumn{2}{|l|}{ Mean } & 4.63 & 4.45 & 12.501 & 1.335 & 13.836 & 6.677 & 7.169 & 6.36 \\
\hline \multicolumn{2}{|c|}{ Solid Sesame } & \multicolumn{2}{|c|}{5.102} & 13.775 & -- & 13.775 & 6.272 & 7.503 & 6.65 \\
\hline \multicolumn{2}{|c|}{ Solid Guar } & \multicolumn{2}{|c|}{13.71} & -- & 4.113 & 4.113 & 2.650 & 1.563 & 0.66 \\
\hline
\end{tabular}

\section{-Total returns and monetary advantage index (MAI):}

The data of economic analysis as influenced by intercropping patterns and the number of cutting of guar compared with solid planting of both crops are presented in Table (5\&6). It reveals that the net return of intercropping $50 \%$ guar and one cut of guar recorded 8.245 and 8.551L.E fad. While the monetary advantage index (MAI) recorded 7.53 and 7.79L.Efadmeanwhile, the lowest net return was recorded for intercropping $100 \%$ guar with sesame and two cuts of guar recorded 1.706 and 3.796L.Efad and monetary advantage index (MAI) of 0.77 and 2.91L.Efadin the $1^{\text {st }}$ and $2^{\text {nd }}$ seasons, respectively. Similar results were recorded by Mokadem and ElKaramaity 1993; Abo-Kerishaet al.,2008; Mahdy and El-Said2015.

-Effect of intercropping patterns of sesame with guar on disease incidence of charcoal rot \& wilt disease complex under natural infestation under field conditions during 2016 and 2017 seasons.

Data in Table 7 show a noticeable effect of different plant density of guar with sesame and number of guar cutting on reducing disease incidence of charcoal rot \& wilt disease complex under the causal fungal pathogenic stress in the field. Generally, all intercropping patterns were significantly decreased disease incidence compared to the sole sesame. The intercropping $100 \%$ Sesame $+100 \%$ guar was the best pattern for the reducing incidence of charcoal rot \& wilt disease complex during the two successive seasons, followed by $100 \%$ Sesame $+66.7 \%$ guar and $100 \%$ Sesame $+50 \%$ guar compared to the sole sesame (control). Furthermore, one cut of guar was better than the two cuts of guar during two seasons. The superior treatment in this respect was the combined treatment of $100 \%$ Sesame $+100 \%$ guar and the one cut of guar in the two growing season. 
Table 7.Intercropping of sesame with guar on disease incidence of charcoal rot $\&$ wilt disease complex under natural infestation under field conditions during 2016 and 2017 seasons.

\begin{tabular}{|c|c|c|c|}
\hline \multirow{2}{*}{ Intercropping Pattern } & \multirow{2}{*}{ No of cutting } & \multicolumn{2}{|c|}{ \% of Charcoal \& Wilt Disease Complex } \\
\hline & & 2016 & 2017 \\
\hline \multirow{2}{*}{ Sesame $+100 \%$ guar } & 1cut & 1.61 & 2.29 \\
\hline & 2 cut & 3.22 & 2.74 \\
\hline Mean & & 2.42 & 2.52 \\
\hline \multirow{2}{*}{ Sesame + $66.7 \%$ guar } & 1 cut & 2.07 & 3.68 \\
\hline & 2 cut & 3.91 & 5.66 \\
\hline Mean & & 2.99 & 4.67 \\
\hline \multirow{2}{*}{ Sesame $+\mathbf{5 0 \%}$ guar } & 1cut & 2.53 & 4.43 \\
\hline & 2 cut & 7.59 & 9.24 \\
\hline Mean & & 5.06 & 6.84 \\
\hline \multirow{2}{*}{ Sole sesame } & 1cut & 9.35 & 11.01 \\
\hline & 2 cut & 9.35 & 11.01 \\
\hline \multirow{3}{*}{$\begin{array}{l}\text { Mean } \\
\text { Average of cutting }\end{array}$} & & 9.35 & 11.01 \\
\hline & 1cut & 3.89 & 5.35 \\
\hline & 2 cut & 6.02 & 7.16 \\
\hline \multirow{3}{*}{ LSD 5\% } & $\mathrm{A}$ & 0.86 & 1.01 \\
\hline & B & 0.61 & 0.72 \\
\hline & $\mathrm{AXB}$ & 1.22 & 1.43 \\
\hline
\end{tabular}

\section{II-Greenhouse experiment:}

Effect of intercropping patterns of Sesame with Guar on disease incidence of charcoal rot, wilt and charcoal rot $\&$ wilt disease complex under artificial infestation with the causal pathogens under pot experiments:

Four intercropping patterns i.e., sole sesame, $100 \%$ sesame $+100 \%$ guar, $100 \%$ sesame $+65 \%$ guar and $100 \%$ sesame $+50 \%$ guar, respectively were used in this study for controlling the incidence of charcoal rot, wilt and charcoal rot \& wilt disease complex caused by phytopathgenic fungi Macrophomina phaseolina and/or Fusarium oxysporum under artificial infestation with the causal pathogens under pot experiments. Results in Table 8 illustrate that, all intercropping patterns were significantly decreased disease incidence of pre- and post- emergence damping off, charcoal rot and/or wilt diseases compared to the sole sesame. $100 \%$ Sesame $+100 \%$ guar was the best intercropping pattern for reducing the incidence of the tested diseases and gave the highest healthy plants of sesame, followed by $100 \%$ Sesame $+66.7 \%$ guar and $100 \%$ Sesame $+50 \%$ guar compared to the sole sesame (control).A mixture of the two phytopathogenic fungi $(M$. phaseolina $+F$. oxysporum) the causal agents of charcoal rot \& wilt disease complex) gave the highest incidence of disease criteria and the lowest \% of healthy plants followed by the causal pathogen of charcoal rot disease (M. phaseolina). Meanwhile, the causal pathogen of wilt disease ( $F$. oxysporum) caused the lowest incidence of disease criteria in this regard. Intercropping guar with sesame can be used for protection of damping-off, charcoal rot and wilt diseases, in this regard this applicable method can significantly contribute to minimize the extensive use of chemical fungicides and consequently reduce their negative impact on both the human beings and the environment as well. This is consistent with the findings of Montaser and kamal 2012. Intercropping sesame with groundnut is more profitable to farmers than groundnut sole planting. The lowest rate of nitrogen fertilization $(107.1 \mathrm{~kg} \mathrm{~N} / \mathrm{ha})$ was the most effective fertilization rate to reduce the proportion of infected plants by the diseases (root - rot and wilt) for both crops under 3:1 intercropping pattern. (AbdelGalil and Abdel-Ghany 2014). The nutritional stress of many plants leads to many diseases, in addition to genetic factors and agricultural practices, the most important of which are the different intercropping systems that increase the ability of plants to resist pathogenic fungi in many studies. (Boudreau 2013). Allelopathy is a phenomenon that normally occurs between donor and target organisms by which the host plants, and living microorganisms that persist in the soil can release some natural chemicals (termend as allelochemicals) into the host environment. Those allelochemicals have either a beneficial or negative effect on each other (Mallik, 2008). Different reasons responsible for releasing those functional allelochemicals including, competition for light, water and nutrients that may occur between mixed crops and may reduce the organism yield. Thus the extent of soil microbial diversity is important for maintaining a good quality of agricultural soil. For this reasons, following a intercropping strategy in agriculture is one of the most reliable and effective way to maintain the of sesame area without significant disturbance in its structure plus reduce the percentage of disease incidence for charcoal- rot and wilt diseases. Affected by both the host and/or the soil borne pathogens on some factors; hence, their effect on soil-borne diseases of different crops is complex and often interrelated and affect the host plant negatively and the fungus positively, leading to a significant increase in damping-off and root rot (El-Garhy, 2000). 
Table 8. Intercropping of sesame with guar on disease incidence, wilt and of charcoal rot, wilt and charcoal rot $\&$ wilt disease complex under artificial infestation with the causal pathogens under pot experiment conditions.

\begin{tabular}{llllll}
\hline \multirow{2}{*}{ Pathogenic Fungus } & Intercropping Pattern & \multicolumn{2}{c}{ \% of Damping-off } & \multicolumn{2}{c}{ \% of Survival Plants } \\
\cline { 3 - 6 } & & $\begin{array}{l}\text { Pre- } \\
\text { emergence }\end{array}$ & $\begin{array}{l}\text { Post- } \\
\text { emergence }\end{array}$ & $\begin{array}{l}\text { Charcoal Rot/ } \\
\text { Wilt }\end{array}$ & $\begin{array}{l}\text { Healthy } \\
\text { Plants }\end{array}$ \\
\hline \multirow{3}{*}{$\begin{array}{l}\text { Macrophomina } \\
\text { phaseolina }\end{array}$} & Sesame + 100\% guar & 7.5 & 12.5 & 12.5 & 67.5 \\
& Sesame + 65\% guar & 10.0 & 15.0 & 17.5 & 57.5 \\
Mean & Sesame + 50\% guar & 15.0 & 15.0 & 20.0 & 50.0 \\
& Control (Sole sesame) & 17.5 & 20.0 & 27.5 & 35.0 \\
Fusarium & & 12.5 & 15.6 & 19.4 & 52.5 \\
oxysporum & Sesame + 100\% guar & 7.5 & 7.5 & 10.0 & 75.0 \\
& Sesame + 65\% guar & 10.0 & 10.0 & 12.5 & 67.5 \\
Mean & Sesame + 50\% guar & 12.5 & 15.0 & 17.5 & 55.0 \\
Mixture of & Control (Sole sesame) & 17.5 & 17.5 & 25.0 & 40.0 \\
M. phaseolina and & Sesame + 100\% guar & 11.9 & 12.5 & 16.3 & 59.4 \\
F. oxysporum & Sesame + 50\% guar & 15.0 & 12.5 & 15.0 & 62.5 \\
Mean & Control (Sole sesame) & 22.5 & 17.5 & 17.5 & 50.0 \\
Average of & & 16.9 & 17.5 & 22.5 & 40.0 \\
Intercropping & Sesame + 100\% guar & 8.3 & 17.5 & 30.0 & 25.0 \\
Pattern & Sesame + 65\% guar & 11.7 & 14.5 & 21.3 & 44.4 \\
& Sesame + 50\% guar & 15.8 & 15.8 & 12.5 & 68.3 \\
& Control (Sole sesame) & 19.2 & 20.0 & 20.0 & 58.3 \\
LSD 5\% for & & & & 27.5 & 48.3 \\
& & 2.75 & 2.48 & 2.24 & 33.3 \\
\hline
\end{tabular}

In addition to preceding and intercropping crops can be used for protection of damping-off and root rot diseases and this method can contribute to minimizing the risk and hazard of toxic fungicides. (Montaser and Kamal 2012). Generally, rotation and intercropping with non hosts is practiced to reduce disease incidence in a subsequent host crop (Baker and Chet, 1982).

The effect of intercropping and proceeding crops on the reduction of damping-off charcoal rot and/or wilt diseasesof sesame plants may be due to the impact of the root exudates of guar plants on the growth of pathogenic fungi in the soil. Several investigators (Mohamed, 1990; El-Garhy, 2000; Kumar et al., 1999) worked on other crops. They found a positive relationship between the susceptibility to the disease and the percentage of spore germination and fungal growth. Root exudates are supposed to play a key role in determining the positive or negative outcome of interaction in the rhizosphere(Bertinet al., 2003; Baiset al., 2006). Different reports indicated that the effect of intercropping and proceeding crops on the reduction of damping-off charcoal rot and/or wilt diseases of sesame plants pointing to the effect of root metabolites or allelochemicals secreted by the neighboring plants on the growth of pathogenic fungi in the soil (Mohamed, 1990; El-Garhy, 2000; Kumar et al., 1999) worked on other crops. Interestingly, those reports showed that root exudates are supposed to play a core role in determining the positive or negative outcome of interaction between the organisms in their rhizosphere (Bertin et al., 2003; Bais et al., 2006).

\section{Conclusion}

Sesame- legumes intercrops produced greater seed yield than either crop grown alone and advantages were shown in land-use efficiency expressed as greater LER than sole crops especially were one cut of guar after 60 days of seeding. Also intercropping guar with sesame significantly reduced the incidence of charcoal rot and wilt disease complex; this is due to the effect of guar root secretions on charcoal rot and wilt pathogens in the soil throughout the growth period of sesame, which led to an increase in the sesame final seed yield. Intercropping of legume fodder crop-- guar with sesame showed money benefits, the guar is a legume fodder crop that is fertile from the soil and provided animal feed in the summer period.

\section{Recommendation:}

Intercrop sesame and guar and one cut of guar after 60 days of seeding was useful for reducing the incidence charcoal rot and wilt disease complex and increased of both sesame and guar seed yield. So that, the pattern of sesame-guar intercrop was recommended for sesame planting that is more profitable for the farmer. 


\section{References}

Abdel-Galil, M. and R. E. A. Abdel-Ghany(2014). Effect of Groundnut - Sesame Intercropping and Nitrogen Fertilizer on Yield, Yield Components and Infection of Root - Rot and Wilt Diseases. International Journal of Plant \& Soil Science 3(6): 623-643.

Abou-Kerisha,M.A.;R.A.Gadallahand E.E.A.Mohamdain(2008). Response of groundnut to intercropping with some sesame varieties under deferent plant density.ArabUniv. J. Agric.,Sci.,Ain Shams Univ.,Cairo.16(2):359375.

Adetiloye,P.O. ; F.O.C.Ezedinma and B.N. Okigbo (1983).A Land equivalent coefficient concept for the evaluation of competitive and productive interactions on simple complex mixtures. Ecol.Modelling., 19:27-39.

Alexander W.L., Bucks D.A. and R.A. Backhaus (1988). Irrigation watermanagement for guar seed production, Agron. J. 80, 447-453.

Agriculture Statistical (2016). Winter Crops. Agriculture Statistical and Economic Sector.1 st Ed., Ministry of Agriculture And Land Reclamation, Egypt.

Agriculture Statistical (2017). Summer \& Nile Crops. Agriculture Statistical and Economic Sector.2nd Ed., Ministry of Agriculture And Land Reclamation, Egypt.

Amanullah J., A. (2017).Intercropping and Rows Configuration Influence Productivity of Dryland Groundnut(Arachis hypogea L.). The International j.,53(2): 92-106.

Bais, H.P.; T.L. Weir ; L.G.Perry;S. Gilroy andJ.M.Vivanco(2006). The role of root exudates in rhizosphere interactions with plants and other organisms. Ann. Rev.PlantBiolo 57: 233-266.

Baker, R. and I. Chet(1982). In: Schneider, R.W.(Ed.), Induction of Suppressiveness Page3550 pp in Suppressive Soils and Plant Disease, 16. American Phytopathological Society, St. Paul, MN, p. 88.

Bertin, C., X. Yang and L.A.Weston( 2003). The role of root exudates and allelochemicals in the rhizosphere. Plant Soil 256: 67-83.

Boudreau MA.(2013). Diseases in intercropping systems. Ann. Rev. Phytopathology.,51:499-519.

El-Fiki, A.I.I.; El-Deeb, A.A.; Mohamed, F.G. and M.M.A. Khalifa (2004). Controlling sesame charcoal rot incited by Macrophomina phaseolina under field condition by using the resistant cultivars and some seed and soil treatments . Egypt.J.Phytopathol.,32 (1-2):103-118

El-Garhy, A.M.M., (2000). Pathological studies on fungal rot diseases on lentil. Ph. D.thesis, Fac. Agric., Al-Azhar Univ.

El-Shennawy, R.Z., Omran, M.M., F.A.A., ElMotteleb(2010). Effect of phosphorus fertilizer treatments on incidence of Fusarium root-rot/wilt disease complex, and on yield components of lupine, chickpea and lentil crops. Arab Univ. J. Agric.Sci. 18: 193-202.

Freed, R.D. (1991).MSTATC Microcomputer Statistical Program. Michigan State -Univ., East Lansing, Michigan, USA.

Gebregergis, and M. , Amare (2019).Effect of Nitrogen Fertilization on the Growth and Seed Yield of Sesame (Sesamumindicum L.). International J., of Agron.,24(2): 254-261.

Ghosh ,P.K. (2004). Comparing growth, yield, competition and economics of groundnut/cereal fodder intercropping systems in the semi-arid tropics of India. Field Crop Research.88(2-3): 227237.

Khalifa, M. M. A.; Abd-El-Megid and Draz, E. I. (2007).Applying some chemical effectors for inducing systemic resistance against sesame charcoal rot disease in Egypt. Egypt .J. of Appl. Sci., 22 (10B): 431-446.

Khalifa, M. M. A.; M. M. Ibrahim and A. A. AbdEl-Baky (2011).Induced systemic resistance against Fusarium wilt disease of sesame by some chemical inducers. Proc.12th Egypt. Phytpathol. Conf., Cario, 3-4 May. Egypt. J. Phytopathol39, (1), _ pp. 24-39.

Khalifa, M. M. A. (1997). Studies of root-rot and wilt diseases of sesame plants. M.Sc.Thesis, Fac. Agric., Moshtohor, Zagazig Univ. Benha Branch, pp. 158 .

Khalifa, M. M. A. (2003). Pathological Studies On Charcoal Rot Disease Of Sesame. Ph. D. .Thesis, Fac. Agric., Moshtohor, Zagazig Univ. Benha Branch, pp.295.

Kumar, S., K. Sivasithamparam ; J.S. Gill andM.W.Sweetingham(1999). Temperature and water potential effects on growth and pathogenicity of Rhizoctoniasolani Anastomosis Group 11 to lupin. Can. J. Microbiol. 45: 389-395.

Mahdy,A.Y.andM.A.A.EI-Said.(2015).Response of sesame for intercropping with some forage crops. Minia J. of Agric. Res. \& Develop., 1(35):139157.

Mallik, U.A. (2008).Allelopathy: advances, challenges and opportunities. In: Zeng, R.S.; Mallik, A.U.; Luo, S.M. (Ed.). Allelopathy in sustainable agriculture and forestry. New York: Springer Sci., Business Midia, p. 25 - 38.

Mc-Gilchrist, C. A. (1965). Analysis of competition experiments. Biometrics, 21: 975-985. (C. F. Field Crop Abst. , 32 (1): 5-6.

Mohamed, M.S., (1990). Effect of cultivating certain winter crops preceding maize in different soil textures on white rot biological control. Assiut J. Agric. Sci. 21:271-281.

Mokadem,SH.A. and A.E.El-Karamity.(1993).The potentiality of intercropping soybean with unbranchedsesame.MinaJ.Agric.,Res.\&Dev.,15(2) :431-452. 
Montaser F. Abdel-Monaimand K. Abo-Elyousr (2012). Effect of preceding and intercropping crops on suppression of lentil damping-off and root rot disease in New Valley e Egypt. Crop Protection 32 : 41-46.

Morsy, K.M.M., (2005). Induced resistance against damping-off, root rot and wilt diseases of lentil. Egypt. J. Phytopathol. 33: 53-63.

MSTAT-C, (1991). A Software Program for the Design, Management and Analysis of Agronomic Research Experiments. Michigan State University, p. 400.

Nassef, D. M.T. and M.A. Abd El-Gaid (2012).Evaluation of yield and its components of intercropped tomato- garlic in New Valley Governorate.Res.J.Agric. \& Biol. Sci.,8:256-260.

Oyeogbe A.; O. Ranti; S. Vaghela and B. Patel (2015). Towards sustainable intensification of sesame-based cropping system versification north western India. J. of Food security Vol. $3: 1-5$.

Puste A. M.; T. K. Mandal; S. K. Gunri; T. S. Devi and B. R. Pramanik (2014). Growth, yield and advantages of green gram - sesame intercropping under different moisture regimes in new alluvial zone of west Bengla. J. of Crop and Well .10 (1) : $19-21$
Rangkadilok, N., Pholphana, N., Mahidol, C., Wongyai, W., Saengsooksree, K.,Nookabkaew, S. and Satayavivad, J. (2010). Variation of sesamin, sesamolin and tocopherols in sesame (Sesamum indicum L.) seeds and oil products in Thailand. Food Chem., 122(3): 724-730.

Shabana, R., A.A. Abd El-Mohsen, M. M. A. Khalifa and A. A. Saber,(2014). Quantification of resistance of F6 sesame elite lines against Charcoal-rot and Fusarium wilt diseases. Adv. Agric. Biol. 1 (3): 144-150.

Steel, R. G. D. and J. H. Torrie (1980). Principle and procedures of statistics, a biometrical approach $\mathrm{Mc}$ Grow-Hill Book Company- Second Edit.

Wafaa, Kh.Mohammed, Nagwa R. Ahmed and W.M. Abdl-El-Hakim.(2013): Effect of intercropping, dates of sowing and $\mathrm{N}$ fertilizers on growth and yield of maize and tomato. Egypt. J. of Appl.Sci., 28(12B):625-644.

Willey, R. W. (1979). Intercropping, its importance and research needs. Part 1, Competition and yield advantages. Field crop Abst. , 32 (1) 1-10.

Willey, R.W. and M.R. Rao (1980): Competitive ratio for quantifying competition between intercrops. Exp.Agric. 16:117-125. 


\section{تأثير تحميل الجوار(محصول علف بقولى) على السمس للحد من الاصابة بمرض العفن الفحمي و الذبول وتأثير ذلك علي المحصول ومكوناته لكلا المحصولين \\ نجوى رفعت أحمد (1)-- محمد محمود أحمد إبراهيم(2) \\ (1)فتم بحوث التنكيف الحصصولى - معهد بحوث المحاصيل الحقلية - مركز البحوث الرزاعية - الجيزة - مصر إير

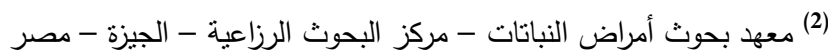

Corresponding author:drnagwarefat2014@gmail.com

تم إجراءهذه الدراسة تحت ظروف الحقل بمحطة بحوث ملوي - مركز البحوث الزراعية بمحافظة الهنيا، خلال موسمين صيفيين متعاقبين 2016 و

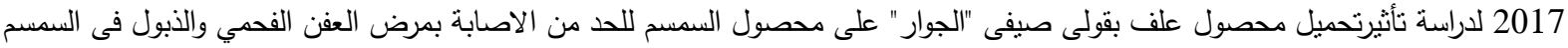
صنف شندويل 3 وتأثير ذلك على المحصول ومكوناتة وعلى العلاقات التنافسية بينهما ـ التهال

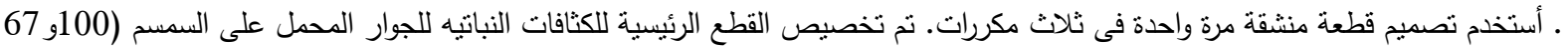
و 50\%) من الموصى به للجوار .والقطع المنشقة لعدد حشات محصول الجوار (مرة- مرتين ).

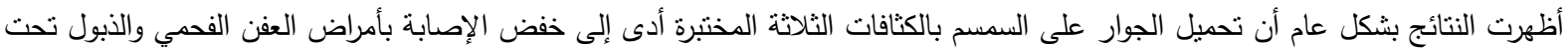
ظروف العدوى الطبيعية بالحقل والعدوى الصناعية بالأصص بالفئ الفطريات الممرضة. - - أظهر نظام التحميل 100\% سمسم إلى 100\% جوار أعلى فاعلية في خفض الإصابة بالأمراض المختبرة.

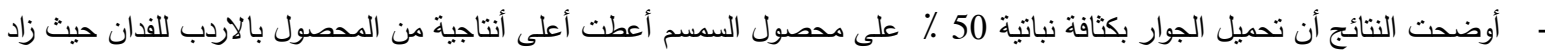

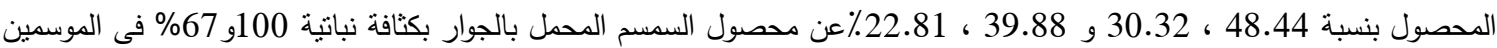

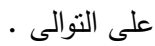
ــ أعلى قيمة للمحصول الطازج والمحصول الجاف (طن/ فدان ) من الجوارالمحمل على السمسم عند كثافة نباتية 100\% من الجوار المحمل

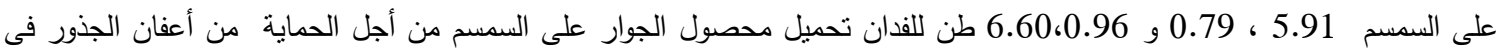
السمسم ، ويمكن أن تسهم هذه الطريقة في تقليل الذبول ومخاطر مبيدات الفطريات السامة.

- سجلت زراعة الجوار بنسبة 50٪ مع السمسم أعلى القيم لمعدل أستغلال الارض والتى بلغت 1.41 و 1.31 و 1.31 في الموسمين على التوالي.

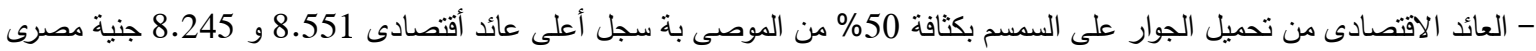
في الموسمين المتتاليين على التوالي. - أوصت الدراسة أنه بمكن تحميل الجوار على السمسم بنسبة 50\%( زراعة الجوار على مسافة 60 سم بين الجور) من الموصى بة وحش

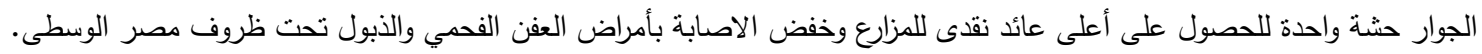

